\title{
Analysis of Behaviour of Phase S Capacitive Tap Insulator of Interconnection 1,220 kV, in Jinamar Power Plant (Island of Gran Canaria, Canary Islands, Spain)
}

\author{
José Manuel García Muñoz ${ }^{1}$, José Antonio Torres Santana ${ }^{2}$ and Miguel Martínez Melgarejo ${ }^{3}$ \\ 1. Endesa, S.A, Las Palmas de Gran Canaria 35008, Spain \\ 2. Edificio de Ingenierías, Departamento de Ingeniería Electrónica y Automática, University of Las Palmas de Gran Canaria \\ (ULPGC) Las Palmas de Gran Canaria35017, Spain \\ 3. Edificio de Ingenierías, Departamento de Ingeniería Eléctrica, University of Las Palmas de Gran Canaria (ULPGC), Las Palmas \\ de Gran Canaria 35017, Spain
}

Received: February 01, 2013 / Accepted: March 04, 2013 / Published: June 25, 2013.

\begin{abstract}
In the island of Gran Canaria, Canary Islands, there are two power plants called Jinamar power plant and Barranco de Tirajana Power Plant, both property of Unelco Generacion, a subsidiary of Endesa Generacion. The first one is placed in the northeast of the island, near its capital, Las Palmas de Gran Canaria, and the other is in the southeast, near the most significant touristic area of the island. Both plants are interconnected by means of four transmission lines, two with a voltage of $220 \mathrm{kV}$ and the other two with $66 \mathrm{kV}$. Since its commissioning in 1995, several phase to ground short circuits have occurred in one of the capacitive tap insulators in the $220 \mathrm{kV}$ lines, in Jinamar side, without a clear cause for them, causing problems of different kind in the electrical system of Gran Canaria. From construction of tap insulators to environmental conditions, every possible factor was carefully studied and laboratory tests were made. In this paper, it is described the analysis which was carried out and how a deep study of every possible cause drove to a definitive solution of the problem.
\end{abstract}

Key words: Tap insulator, capacitive, dirtiness, shortcircuit, field tests.

\section{Introduction}

In the island of Gran Canaria, Canary Islands, there are two power plants called Jinamar Power Plant and Barranco de Tirajana Power Plant, both property of Unelco Generacion, a subsidiary of Endesa Generacion. The first one is placed in the northeast of the island, near its capital, Las Palmas de Gran Canaria, and the other is in the southeast, near the most significant touristic area of the island. Jinamar Power Plant has an installed capacity of 416 MW, and Barranco de Tirajana Power Plant has 697 MW. Both plants have practically every thermal generation

Corresponding author: José Manuel García Muñoz, engineer, research fields:operation \& maintenance of electric systems. E-mail: josemanuel.garciam@endesa.es. technology (classic steam cycle, combined cycle, gas turbines and Diesel generators) and there is even a small wind plant in the second one. Both plants are interconnected by means of four transmission lines, two with a voltage of $220 \mathrm{kV}$ and the other two with $66 \mathrm{kV}$. These two last have several intermediate substations inserted along their traces, all of them being of interior conventional type. Both $220 \mathrm{kV}$ lines go straight from one plant substation to the other, and both of them are built totally with SF6 technology, having a length of $40 \mathrm{~km}$ each, being arranged in a double circuit line. Today, all of them are owned by Red Electrica de España, but formerly were by Endesa Distribucion Electrica. Both $220 \mathrm{kV}$ substations, Barranco de Tirajana Power Plant and both 220 kV 
interconnection lines were put in service in 1995. Since then, with a certain regularity, several one phase to ground short circuits have been occurring, with their origin in the capacitive tap insulator installed in the central phase (S phase) in the outlet of the so called interconnection I line in Jinamar substation. In general, these situations are solved through the action of corresponding protective relays. Nevertheless, in some occasion, and with certain operational conditions in the system, some unbalancing between generation and demand has occurred, in such a way, the integrity of the whole electric system has been in serious danger. In the diversity of tries to get a solution for this problem, several measures were taken. From operational actuations such as intensification of cleanings, to the mere change of the insulator with a certain periodicity (every 3-4 years), passing by constructive alterations such as modification of insulator head, all of them have proved to be ineffective and did not produce any improvement in the situation. In 2005, after last incident occurred, a decision was taken by the company who had the property of installations, Endesa Distribucion Electrica, S.A.. This decision was to carry out an exhaustive study of possible causes which were provoking these short circuits. As the number of insulators of this type installed in the Canary Islands is 24 and only the one installed in phase S of the so called interconnection I line in Jinamar substation produced problems, such failures had to do with the environment becauseafter every failure, the line returned to service again with the same insulator in all cases.

\section{Results of Bushing Inspection and Records of Incident}

Following the last incident, in May 20, 2005, it began a detailed investigation of all factors involved in it, including the whole protective system [1]. This analysis showed that in all cases through 10 years in service, defects were initiated when, in its evolution, voltage reached its maximum value. It was also revealed that at the beginning of the short circuit, sane phases were affected due to electrostatic capacitive coupling between them. In addition, the fault homopolar current had a very high amplitude.With this information, the obvious conclusion was that in the bushing there was an area where,in some way, the level of allowed dielectric strength was reached and that the high level of current had to leave, necessarily, traces of its passage. Therefore, the challenge was to discover the factor that produced such a level of rigidity necessary for the occurrence of short circuit. It should be noted here that the level of isolation in the bushings corresponds to $380 \mathrm{kV}$, given the special environment of salinity in the area. After making an inspection of the insulator (Fig. 1), next deductions could be obtained:

- From first to last bell there were no signs of an outer arc. This whole area was spotless;

- The upper surface of the first bell had not suffered, but between the surface of the underside of the same bell, and the principle of the flange, it could be seen that these areas were subject to a lot of heat. This heat was due to current flow and generated by Joule effect;

- The defect was internal.

\section{Capacitive Type Bushings}

For voltages above $100 \mathrm{kV}$ capacitive isolators are used because they achieve a more evenly distribution of the voltage gradient across the thickness of the insulation. Capacitive isolator consist schematically of insulating substance several tubes of different heights and equal thickness separated from each other by very thin metal tubes. The whole is equivalent to a certain number of elementary capacitors in series. Therefore, if the capacitor formed by each pair of leaves and the dielectric cylinder between themhave equal capacity, none of the insulating tubes will be subjected to a voltage greater than $V / n$, being " $n$ " the number of elements. As the capacity of a cylindrical capacitor increases with the length and radius of the cylinder, 

in Jinamar Power Plant (Island of Gran Canaria, Canary Islands, Spain)

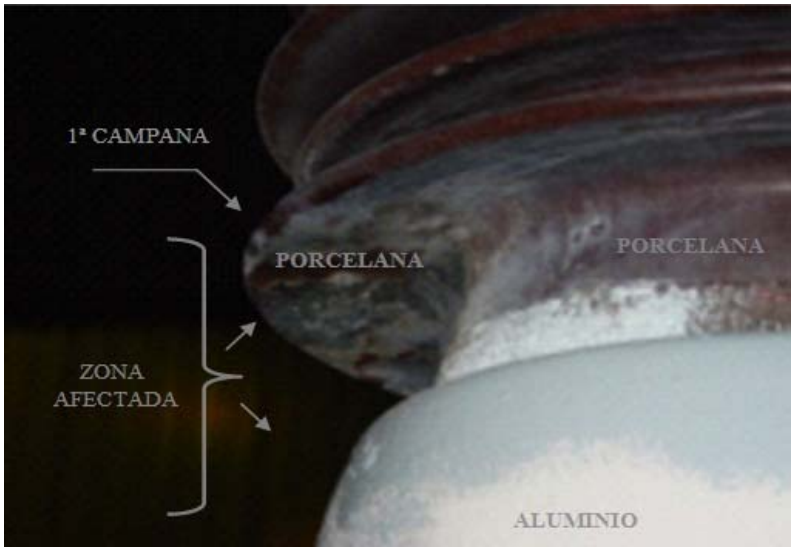

Fig. 1 There are no other external findings shedding light on the ultimate cause of the incident. Efforts were directed to simulate the environmental conditions of operation.

the length of the tubes must be decreasing with increasing the diameter thereof. These capacitors must be built with special care, using well homogeneous insulators and ensuring that the smaller diameter pipe insulation is securely attached to the central conductor, without any trace of air [2]. According to standards IEEE-C57.19.00 and C57-19-01,capacitive insulators or bushings for $115 \mathrm{kV}$ or higher are formed by a capacity C1 for the main insulation, and a second derivation capacity known as C2. The capacitor C1 represents the total equivalent capacity of elementary capacitors in series made each one with paper impregnated with oil or resin, and a metal screen (metal cylinder). They are built in a process repeated to form the equivalent C1 capacitor.The capacitor is formed by the outermost metal cylinder, which is rigidly attached to ground through the flange (Fig. 2).

The outer layer is attached to a terminal ("Voltage Tap Stud” in Fig. 2), which is accessible externally to carry out capacity measures and the Joule losses factors in both capacitors. Following completion of measuresand prior to commissioning, the outer armor must be grounded simply by turning the screw terminal tests. Through the test terminal, a certain voltage can be obtained when insulator is at service voltage. By suitable devices, the output voltage can beused to operate relays and other measurement and control modules. The output terminal can also be used to monitor partial discharges during factory testing. It

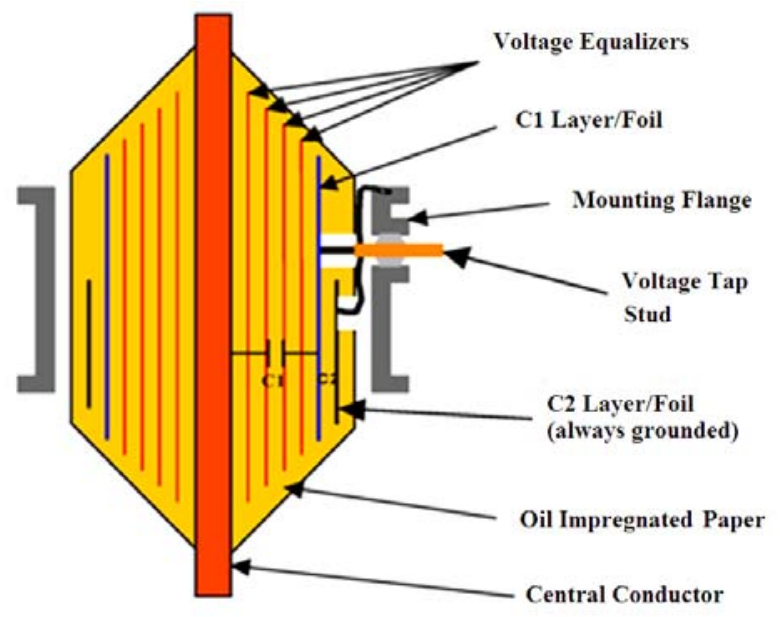

Fig. 2 Internal structure of capacitive bushings.

must be taken into account that power factor of $\mathrm{C} 1$ and C2 can be affected by external factors such as contamination of porcelain, especially when it comes to salt contamination, air and oil cooling if one exists [3].

\section{Capacitive Bushings Located in the S-Phase Line Interconnection I}

This is a bushing of capacitive type, built with cylindrical capacitorswith a dielectric formed by special paper impregnated with epoxy resin.For measurements of power factor and capacity, it has a hex head screw, protected against dust and moisture. The test voltage applied during the tests at the factory is $2 \mathrm{kV}$ with a frequency of $50 \mathrm{~Hz}$ during one minute. This means that at the time of testing it should not be applied a voltage higher to $500 \mathrm{~V}$ because a partial discharge may occur in the region of the test tap which could affect the measurement. The test tap should not be left open during operation. Once the test made, it must be checked that the spring grounding and protective cover are in the correct position.

\section{Theoretical Interpretation of the Most Likely Cause of Defects [3]}

Measurements may be affected by the support structure, if packing timbers are damp or wet, by resistance between the flange of the insulator and the metal frame for support, isolated effects with other 

in Jinamar Power Plant (Island of Gran Canaria, Canary Islands, Spain)

objects, and external connections during testing.The bushing has a capacity $\mathrm{C} 2$, which depends on other layers of paper with adhesive and insulation level of the tap test. Changes in the other layers of adhesive paper can affect the power factor of several of the same type bushings. Furthermore, the proximity of C1 layer to the flange creates a large electrostatic coupling between both sides. Because of this effect, porcelain, oil and air surrounding bushing, may have some effect on power factor during the measurements. Specifically, there are manufacturers who have bushings that can withstand high currents produced with a short flange and the outer layer for C1 long. This translates into greater coupling between the metal most external layer of $\mathrm{C} 1$ and surrounding materials. This fact drives to a trend of showing high values of power factor during the measurements. Depending on the design, the power factor of C2 in such bushings may be between $0.1 \%$ and $2 \%$. It has been said previously that when measurements are made in the ends of condenser C2 one must be careful to do it with insulator perfectly clean. Otherwise, and depending on the type and degree of external contamination, it is produced an increase of power factor. But when the insulator is put into operation the capacitor C2 is completely short-circuited through the switch or testing screw, obviously remaining condenser $\mathrm{C} 1$ in service. Therefore, what really matters is whether there is any variation of the parameters related to the insulator as go on polluting.Between the screen's outer metal and the flange there are several dielectric materials, which are paper impregnated with oil or epoxy resin, oil and porcelain.When considering different permittivity values for each of these dielectrics, it appears that thoseof lowest permittivity will be the ones that sufferhighest efforts. This problem may be aggravated if it is further assumed that during the manufacturing process there are bubbles of air into the area between adhesives and paper. If so, in the pointswhere there are air bubbles probably will begin partial discharges. Consequently, it is necessary to remove the air inside the insulation occupying all possible gaps for which different procedures are used for impregnation. In the next point it is analyzed how pollution can lead to a situation which provokes initially partial discharges in the bubbles due to increased tension and therefore the gradient at the ends of the elementary capacitors closest to the center conductor (Fig. 3).

If measurement of power factor of $\mathrm{C} 1$ is made with the insulator totally clean at a constant temperature, it is clear that if this situation persists, the power factor will also remain constant. However, if with the measurement bridge connected, the degree of salt contamination at constant temperature is increased, the power factor will be also increased due to formation of new internal capacities, indicated in Fig. 7 by $C_{i}$ and $C_{j}$. These capacities are being left in parallel with the basic screens which are closer to the inner walls of porcelain.

Continuing with Fig. 3, it should be noted that between the flange and the first bell of insulator (looking from the bottom up), there is a transverse contraction that facilitates the creation of these new capacities in function of the level of contamination. Within this area formed by the porcelain, rim and bottom of the hood (fin), is deposited a conductive film which, in contact with the flange, facilitates the

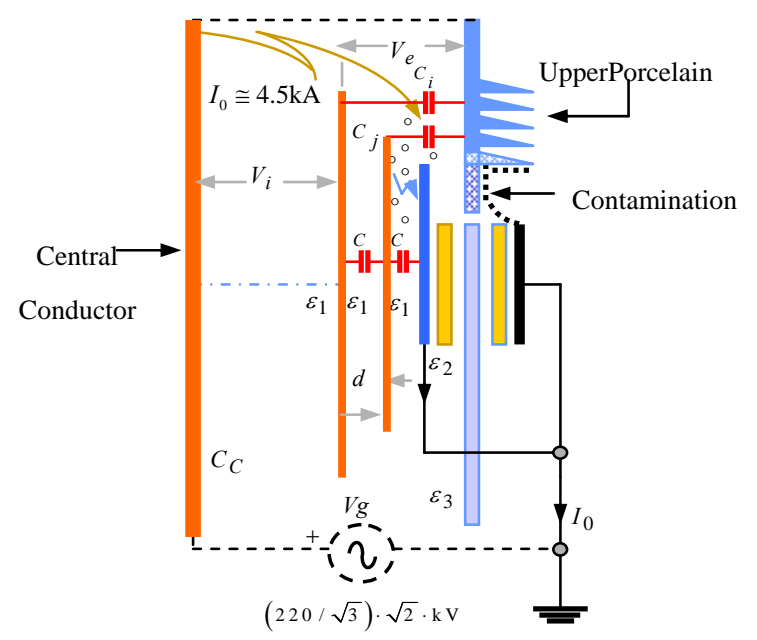

Fig. 3 Partial discharges in the bubbles due to increased voltages. 

in Jinamar Power Plant (Island of Gran Canaria, Canary Islands, Spain)

formation of a new armor. Jinamar substation is close to the sea, which makes the insulator constantly subject to heavy pollution of saline type. Being a salt film, its more or less metallic behaviour depends on degree of hydration in every moment. Furthermore, for the lower hood to be subject to very severe pollution that can lead to discharges, the sense of air has to be bottom up.It is a proven fact that the relative humidity reached in the time interval in which the incidents of this type occur is between $90 \%$ and $97 \%$. The higher hydrating of contaminant, the higher is the mobility of ions, thereby causing successive partial discharge inside the bubbles, combined with increased voltage gradient at the end of the cylindrical capacitors. When thissituation is maintained, the medium is going to ionize increasingly until an electric arc is discharged between the central conductor and the interior ground terminal.

\section{Power Factor Measurements and Capacity}

The power factor is a very important property in capacitive insulators. This factor is mainly determined by the level of moisture contained inthe paper, and the amount of pollutants in the insulation system. Moreover, the power factor depends largely on temperature [4].

Fig. 4 shows the changes experienced by the power factor versus temperature for different degrees of relative humidity in oil impregnated paper bushings.We can see clearly that the slopes of the curves increase at elevated temperatures. The dissipation factor is practically the same for relative humidity values ranging between $0.1 \%$ and $1 \%$ at a temperature of $20{ }^{\circ} \mathrm{C}$, so it is more important to measure or consider the power factor at high temperatures, and not at $20{ }^{\circ} \mathrm{C}$. The bushing under test has a test tap located between capacity $\mathrm{C} 1$ (main insulation) and $\mathrm{C} 2$. The value of the RMS voltage applied to make the measurements was not higher than $2 \mathrm{kV}$, being the same used by the bushing manufacturer (Fig. 5) [5].

If this voltage level is exceeded, a partial discharge may occur in the region of the test tap, affecting the outcome of the measures. To avoid measurement errors due to possible interferences, we proceeded to move the bushing to the testing laboratory RODRITROL Company, Ltd., in Las Palmas de Gran Canaria. The bushing was fastened upright on a bed of iron ground, proceeding to its cleaning with a dielectric solvent.In wet conditions, it is necessary to dry the test tap in order to ensure representative readings of power factor $\mathrm{C} 1$ and $\mathrm{C} 2$. As with other electrical equipment, the test tap may dry with a quartz lamp or an air dryer.On the other hand, efforts were made to the cables used in the measurements which were as short as possible and not be in contact with objects that were connected to ground. For routine testing of new insulators, the ANSI and IEC standards require that the power factor measurement is made at room temperature. By measuring the power factor in insulators with resin impregnated paper (RIP) core, a deviation should be noted regarding the value

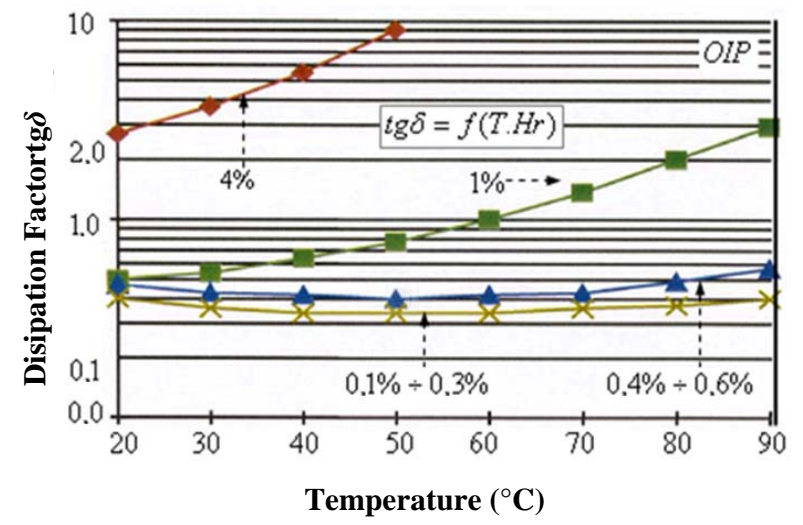

Fig. 4 Disipation factor vs. temperature.

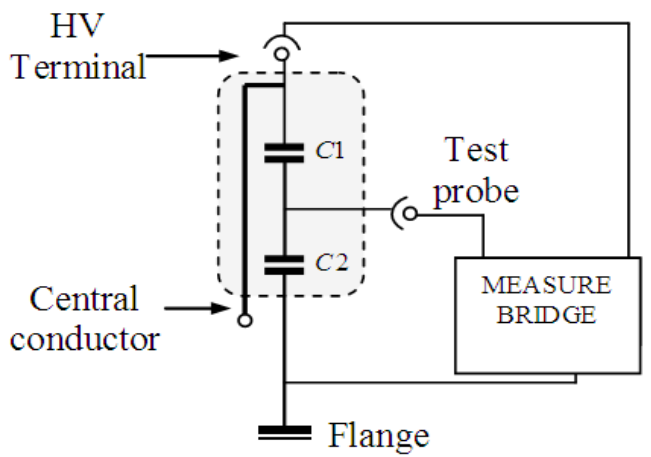

Fig. 5 Scheme of bushing test. 

in Jinamar Power Plant (Island of Gran Canaria, Canary Islands, Spain)

indicated on the nameplate before its commissioning. Probably the reason for this is the penetration of moisture in the surface layer of RIP insulator. This may occur if the insulator is stored without a protective bag sealed, allowing the entry of air with high humidity levels in the outer surface layer of insulator. Generally, if the insulator is stored for a time in a protected area against possible variations in moisture, the value of power factor will very nearly match with that specified by the manufacturer.The measuring bridge used to measure the capacity and power factor $(\tan \delta)$ has been the AVO BIDDLE DELTA 2000 with number 48120403. For insulator resistance it has been the model Megger MEG-10-01 with number 030303/1159 with a maximum voltage for measure of $10 \mathrm{kV}$. With the aforementioned bridge measurements can be made in differential mode, i.e., ungrounded. In this way the influence of parasitic capacitances has little influence on the outcome measures.

\section{Power Factor Variations with Temperature and Humidity}

Measurements were made with the capacitor short-circuited, because it is the usual way to operate these types of insulators.

\subsection{Evolution of the Power Factor as a Function of} Humidity for $\gamma=0.222 \mathrm{mS} / \mathrm{cm}$

In this case, it comes to analyze the evolution of the power factor as a function of relative humidity at constant temperature. This is what is being searched, but in practice the temperature did not remained exactly constant, but approximately constant. Specifically, between $20^{\circ} \mathrm{C}$ and $22^{\circ} \mathrm{C}$.

In Fig. 6, it is shown that power factor increases as it does the relative humidity.

\subsection{Evolution of the Power Factor as a Function of} Humidity for $\gamma=46.7 \mathrm{mS} / \mathrm{cm}$

In this case, we have tried to reproduce the same environmental conditions that normally are under the insulators. To cause variations, the degree of relative humidity has been taken in this case sea water with aconductivity of about $46.7 \mathrm{mS} / \mathrm{cm}$. Making a comparison between both tests the conclusions are that for a value of relative humidity of $40 \%$ power factor value is approximately 0.6 in the case of mineral water used to humidify with a conductivity of $0.222 \mathrm{mS} / \mathrm{cm}$. But for about the same moisture content, but using salt water with a conductivity of 46.7 $\mathrm{mS} / \mathrm{cm}$, the power factor is 0.7 .

\subsection{Evolution of the Power Factor as a Function of Temperature}

In this case, as well as the next one, what has been done has been to measure changes in the power factor while maintaining the relative humidity with a constant value $H_{r}$ of $50 \%$.

\subsection{Evolution of the Power Factor as a Function of Temperature for $\gamma=46.7 \mathrm{mS} / \mathrm{cm}$}

Although, the temperature values are not exactly the same, again comparing tests, reached the following conclusions:

- In the mineral water test power factor remains constant when the temperature is varied from $19.3^{\circ} \mathrm{C}$ to $28^{\circ} \mathrm{C}$;

- The power factor increases as does the temperature to lower values of conductivity;

- From a temperature of $28{ }^{\circ} \mathrm{C}$, the power factor

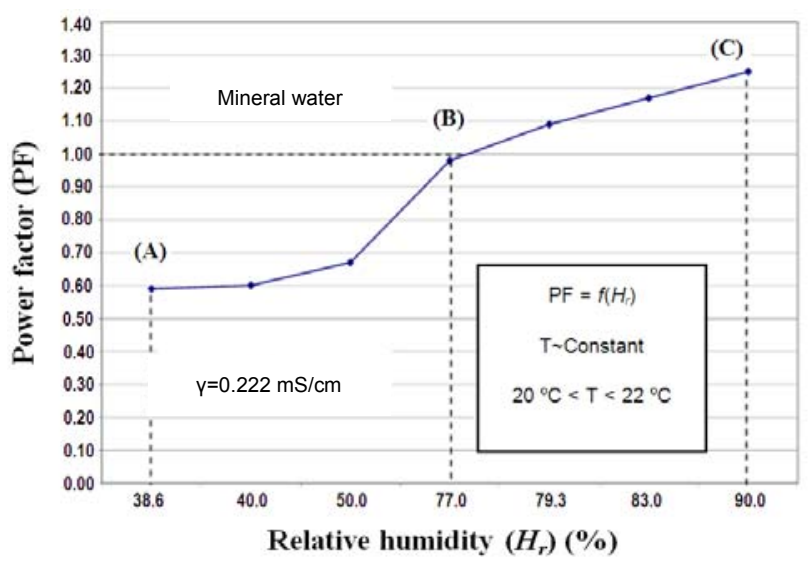

Fig. 6 Power factor vs. relative humidity. 

in Jinamar Power Plant (Island of Gran Canaria, Canary Islands, Spain)

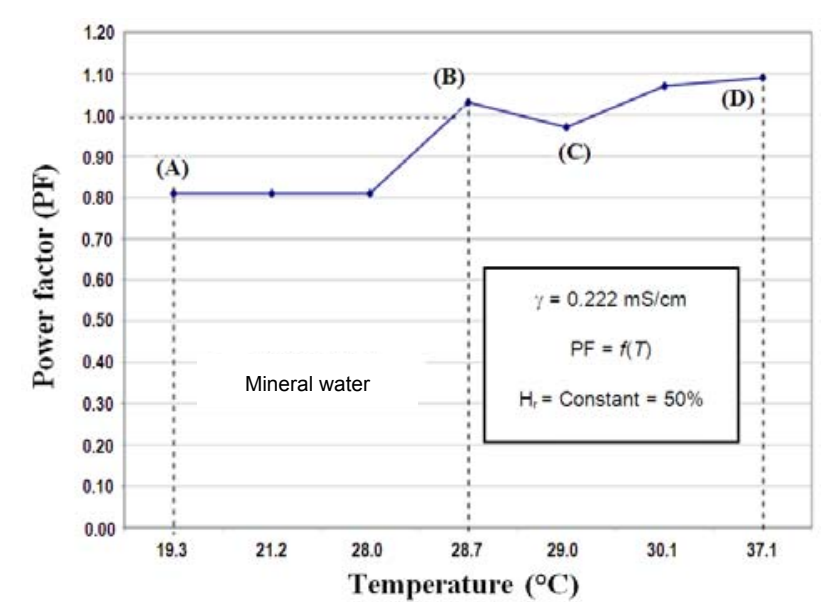

Fig. 7 Power factor vs. temperature.

evolves more uniformly with temperature for the case where the water has a higher conductivity.

These test showed that the power factor is more sensitive to variations and degree of pollution than to changes in temperature.

\section{Variations of Capacity C1 with Humidity and Temperature}

With the capacitor C2 in shortcircuit through the tests switch, in this case it was registered the evolution of capacity C1 for variations in humidity and temperature. As in the previous case, mineral water with a conductivity of $0.222 \mathrm{mS} / \mathrm{cm}$ and sea water with a conductivity of $46.7 \mathrm{mS} / \mathrm{cm}$ were used to wet the insulator.

Humidification was made with mineral water, trying to keep temperature within a range between 20 ${ }^{\circ} \mathrm{C}$ and $22{ }^{\circ} \mathrm{C}$. It was not possible to keep temperature exactly constant as it would have been desirable. For a variation of relative humidity based on mineral water and between $77 \%$ and $90 \%$, it can almost be said thatcapacity varies linearly with temperature. In this case, within the range being considered, the sensitivity is very small: it just passes from $534.38 \mathrm{pF}$ to only 535 PF. Rest of tests made showed that capacity C1 increases with humidity and salinity. As a conclusion of tests it should be noted that, although the presence of salt contamination could influence the appearance of discharges and eventually cause a fault, this influence was not in itself enough to be the determining factor. There should be an additional cause, combined with that, for incidents to occur. Such a cause had to be necessarily, as it was, in the environment of the bushing.

\section{Boundary Conditions}

In addition to the tests described, therefore, it was carried out a detailed analysis of the capacitive bushings environment, making also a comparison with the one in the rest of similar substations, either in Gran Canaria (Barranco de Tirajana) or in Tenerife (Candelaria and Granadilla). Conclusions were:

- Barranco de Tirajana and Granadilla substations have an environment in which insulators are well ventilated and away from abnormal contamination, except for floating dust and moisture which are usually in the area. A differential fact is that 220/66 $\mathrm{kV}$ transformers are in the back of the substations buildings, so that their high temperatures and ventilation cannot influence any thermal shock in the bushings;

- Regarding to Candelaria substation, although the transformers are located on one side of the lines, their ventilation has no influence on the bushings, as wind direction is usually perpendicular to the installation so that any hot air current rising from transformers do not impact on them;

- However, at the substation Jinamar were found the following differences:

First, the bushings are installed on the roof of the substation. The rest of substations are at ground level.

Transformers are just below the bushings of the lines, there being a direct effect of the hot air flow ventilation of transformer bushings number 2 on where the faults occurred.

The prevailing winds in the Canary Islands are the trade winds from the northeast. It can be seen in the picture in Fig. 8 that the degree of deterioration of the paint on the metal structure is greater on that side of the facility, coinciding with the position of the $\mathrm{S}$ phase 


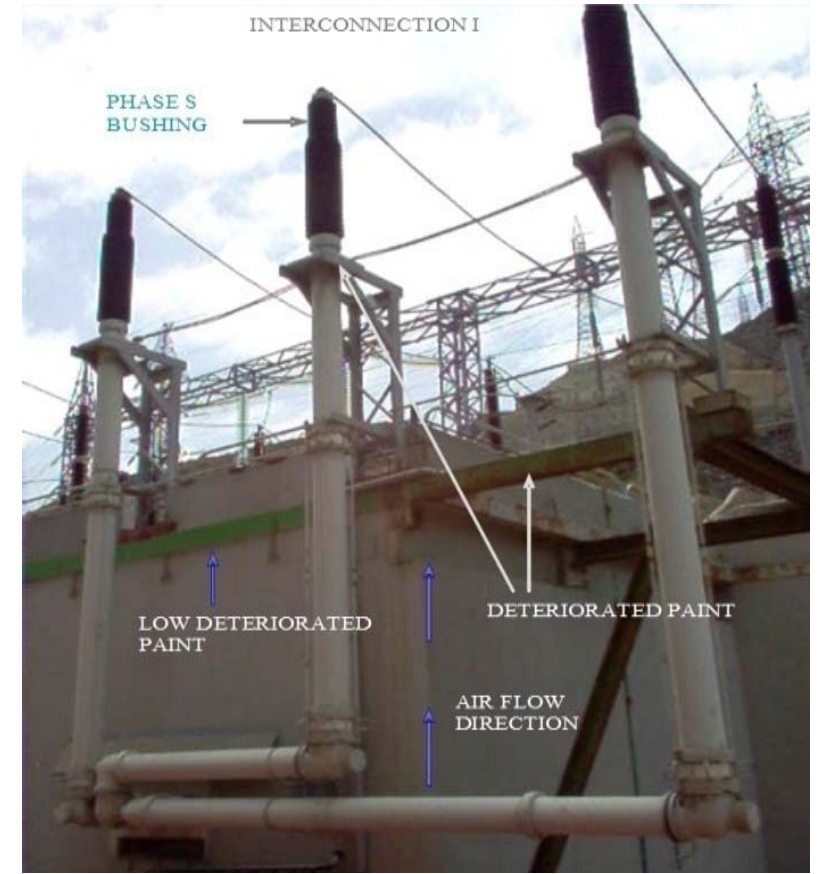

Fig. 8 Picture of installation.

of Line 1. In fact, this painting has historically had touched up more frequently than in the rest of the structure.The inference (obviously non-obvious) is that the hot air generated by the high temperatures of the transformer tended to rise because its density is lower than the cold air, with its speed also increased by fans that are installed on the bottom of the transformer to promote natural ventilation.

The area of transformers was half covered and the non covered half is homeless for ventilation, so warm air, which tended to rise to reach the roof area, must seek out rising in parallel to the wall substation where it also met the hot air from the roof zone, because the prevailing trade winds pushed it against the wall, producing a kind of chimney effect whereby hot air was routed to the bushings of $\mathrm{S}$ phase of line 1 , directly impacting the bottom of the bushing. In circuit 2, being in the same provision, the chimney effect does not occur because the prevailing wind pushes hot air away from the line. This incidence of warm air at the base of bushings created a unique boundary conditions in S phase with a very sharp local increase in temperature which, combined with salt contamination, did significantly increase the power factor determining the appearance of defects and resulting in franc short-circuits to the ground.

\section{Solutions}

Solution adopted to solve the problem was completely roofed enclosure of the transformer, so that hot air would not impact the base of the bushings. In addition, the insulators of each of the phases were equipped with a polymeric insulating varnish in order to reinforce the line leak. Before adopting such measures the normal rate of incidents was more or less one per year.Up to now there have been no more incidents. Moreover, the thermograms that are held regularly have shown no evidence of abnormal temperature in the bushings of the circuit S phase I or anyone else.

\section{Conclusions}

This article shows how the comprehensive investigation of all factors that could influence the recurring incidents since the start of the installation in 1995, including internal analysis equipment of which there was not too much technical information available, combined with a careful observation of physical disposition, allowed to reach a solution that has proven to be the right and final solution of a situation that had been causing many problems during years in the Gran Canaria electric system.

Only the combination of a careful theoretical study of every condition involved in the incidents and the comprehensive analysis of physical conditions of bushings and its whole installation drove to a successful solution of a serious operational problem of $220 \mathrm{kV}$ network in the island of Gran Canaria.

\section{Acknowledgments}

This study was carried out along with University of Las Palmas de Gran Canaria, with the invaluable assistance of the Professor Fernando Garnacho, Director of Central Official Electrotechnic Laboratory, in Madrid, and Professor Luis Rouco, of Comillas 
Pontifical University, whose views and opinions were of great importance in the development of the research carried out. The authors also want to thank the company RODRITROL, based in Las Palmas de Gran Canaria, for its selfless assistance in conducting the tests that were carried out.

\section{References}

[1] Paulino Montané Sangrá, Protections in Electrical Installations, Marcombo, 1988, chapters 3-5.
[2] A. Roth, High Voltage Technology, Labor, SA.

[3] P. Singh, C2 Power Factor and Capacitance of ABB Type O Plus C, AB And Type T Condenser Bushings ABB Inc., USA, 2003, pp. 1-6.

[4] D.X. Zeng, An Improved Method of Measuring Power Factor ofC1-Graded Resistance Bushing, Lapp Insulator Company, LeRoy, NY, pp. 1-7.

[5] Insulators diagnostics and conditioning, leaflet, ABB Publications. 